\title{
THE WIENER INTEGRAL AND PERTURBATION THEORY OF THE SCHRÖDINGER OPERATOR ${ }^{1}$
}

\author{
BY DONALD BABBITT \\ Communicated by Ralph Phillips, October 1, 1963
}

Introduction. It has been known for a long time that the Wiener integral can be effectively used in studying various properties of the Schrödinger operator $\Delta-V(x)$ where $\Delta=\partial^{2} / \partial x_{1}^{2}+\cdots+\partial^{2} / \partial x_{m}^{2}$, the Laplacian in $m$-dimensional Euclidean space $E^{m}$ and $V(x)$ is a realvalued function on $E^{m}$ satisfying certain regularity conditions. For example, see works by Kac [6], Getoor [2; 3], Ray [9] and Nelson [8].

Getoor [2] was the first to point out the connection between a Wiener type integral and the perturbation theory of certain operators of the form $\Omega-V(x)$ where $\Omega$ is the infinitesimal generator of a homogeneous Markov process on a locally compact metric space $X$ and $V(x)$ is a real-valued Borel measurable function on $X$, bounded on bounded sets and bounded below everywhere. When $X=E^{m}$ and the Markov process is a suitably normed Wiener process, ${ }^{2} \Omega=\Delta$ and his results apply to the Schrödinger operator $\Delta-V(x)$.

In this paper we will only consider the case $X=E^{m}$ and $\Omega=\Delta$ but $V(x)$ will be allowed to have certain singularities precluded by Getoor's conditions on $V$. For example, we can consider the perturbation of $\Delta+e / r$, where $X=E^{3}$ and $r=\left(x^{2}+y^{2}+z^{2}\right)^{1 / 2}$, i.e., the Schrödinger operator with attractive Coulomb potential. In particular the essential condition on $V(x)$ is that $\Delta-V$ is semi-bounded above on a certain dense domain $D$ in $L_{2}\left(E^{m}\right)$, the Hilbert space of complexvalued square summable function on $E^{m} .^{3}$

Main results. In this section we will introduce some notation, state our theorems and corollaries, sketch one proof and remark on the remaining proofs.

$C_{0}^{\infty}\left(E^{m}\right)$ will denote the space of infinitely differentiable, complexvalued functions on $E^{m}$ with compact support. Let $L_{2}^{\text {loc }}\left(E^{m}\right)$ denote

\footnotetext{
${ }^{1}$ The preparation of this paper was sponsored in part by the National Science Foundation.

${ }^{2}$ In particular we assume $\sigma^{2}=2$ where $\sigma^{2}$ is the variance per unit of time of the Wiener process. See [6].

${ }^{3}$ Definition. A symmetric operator $A$ with domain $D \subseteq L_{2}\left(E^{m}\right)$ is said to be semibounded from above if there exists a real number $k<\infty$ such that $(A \psi, \psi) \leqq k(\psi, \psi)$ $\equiv k\|\psi\|^{2}$ for all $\psi \in D$ and where $(\phi, \psi)=\int \phi \bar{\psi} d x$.
} 
the space of complex-valued functions on $E^{m}$ which are square summable on every compact subset of $E^{m}$. We will assume as known the definition and properties of a Wiener process on $E^{m}$ and the related integration (Wiener integral) theory. In particular if $F(\cdot)$ is a measurable functional over Wiener space then $E(F(\cdot) \mid x(0)=x)$ will denote the Wiener integral (expectation) of $F$ over the Wiener process which begins at $x$ at time 0 , if the integral exists. ${ }^{4}$

We also assume as known the concept of a set of capacity zero. ${ }^{5}$

We can now state the main results.

THEOREM 1. Let $V(x)$ be a real-valued function on $E^{m}$ which is in $L_{2}^{\text {loc }}\left(E^{m}\right)$ and is continuous except for a set $Q$ of capacity zero. Suppose further that

$$
((\Delta-V) \psi, \psi) \leqq k(\psi, \psi)
$$

for $\psi \in C_{0}^{\infty}\left(E^{m}\right)$ and $k<\infty$, independent of $\psi$. Then, for $\psi \in L_{2}\left(E^{m}\right)$,

$$
\left(T_{t}^{V} \psi\right)(x) \equiv E\left(\exp \left[-\int_{0}^{t} V(x(\tau)) d \tau\right] \psi(x(t)) \mid x(0)=x\right)
$$

exists and defines a strongly continuous self-adjoint semi-group of operators on $L_{2}\left(E^{m}\right)$ whose infinitesimal generator is a self-adjoint extension $\Delta-V$ considered as an operator on $C_{0}^{\infty}\left(E^{m}\right)$.

SKETCH OF PROOF. Let

$$
V_{N}(x)= \begin{cases}V(x) & \text { if } V(x)>-N, \\ -N & \text { if } V(x) \leqq-N\end{cases}
$$

which is in $L_{2}^{\text {loc }}\left(E^{m}\right)$ and is continuous except for a set of capacity zero. It then follows from a theorem of Getoor [2] and the general theory of self-adjoint semi-groups $[4, \S 22.3]$ that $T_{t}^{V_{N}}$ is a strongly continuous self-adjoint semi-group on $L_{2}\left(E^{m}\right)$ whose infinitesimal generator is the self-adjoint extension of $\Delta-V_{N}$ considered as a symmetric operator on $C_{0}^{\infty}\left(E^{m}\right) \cdot{ }^{6}$ Since

$$
\left(\left(\Delta-V_{N}\right) \psi, \psi\right) \leqq((\Delta-V) \psi, \psi) \leqq k(\psi, \psi)
$$

the spectrum of the self-adjoint extension of $\Delta-V_{N}$ is $\leqq k$ and thus from the spectral representation of $T_{t}^{V_{N}}$ we have

\footnotetext{
${ }^{4}$ See Kac [6], Getoor [2] and Nelson [8] for a pertinent discussion of Wiener space, Wiener integral, etc.

${ }^{5}$ See Nelson [8] for a brief discussion of this subject.

${ }^{6}$ Results of Ikebe and Kato [5] assure us that $\Delta-V_{N}$ is essentially self-adjoint with domain $C^{\infty}\left(E^{m}\right)$.
} 


$$
\left\|T_{t}^{V_{N}}\right\|_{\mathrm{op}} \leqq e^{k t}
$$

where \|\|$_{o p}$ is the standard operator norm.

Moreover for $\psi \in L_{2}\left(E^{m}\right), x \notin Q$, we have by the monotone convergence theorem that

$$
\begin{aligned}
\left(T_{t}^{V_{N}}|\psi|\right)(x) & =E\left(\exp \left[-\int_{0}^{t} V_{N}(x(\tau)) d \tau\right]|\psi(x(t))| \mid x(0)=x\right) \\
& \uparrow E\left(\exp \left[-\int_{0}^{t} V(x(\tau)) d \tau\right]|\psi(x(t))| \mid x(0)=x\right) \\
& =\left(T_{t}^{V}|\psi|\right)(x)
\end{aligned}
$$

where the convergence is pointwise (in $x$ ). Since a set of capacity zero has measure zero, we have pointwise almost everywhere convergence of $T_{t}^{V_{N}}|\psi|$ to $T_{t}^{V}|\psi|$. Thus

$$
\left[\left(T_{t}^{V_{N}}|\psi|\right)(x)\right]^{2} \uparrow\left[\left(T_{t}^{V}|\psi|\right)(x)\right]^{2}
$$

almost everywhere and from (1) we have

$$
\int\left[\left(T_{t}^{V_{N}}|\psi|\right)(x)\right]^{2} d x \uparrow \int\left[T_{t}^{V}|\psi|(x)\right]^{2} d x \leqq e^{2 k t} \cdot\|\psi\|^{2} .
$$

From this we conclude that $T_{t}^{V}|\psi| \in L_{2}\left(E^{m}\right)$ and hence $T_{t}^{V} \psi \in L_{2}\left(E^{m}\right)$. From the definition of $T_{t}^{V_{N}}$ and $T_{t}^{V}$ we see that

$$
\left|\left(T_{t}^{V_{N}} \psi\right)(x)-\left(T_{t}^{V} \psi\right)(x)\right|^{2} \leqq 4\left[\left(T_{t}^{V}|\psi|\right)(x)\right]^{2}
$$

and thus

$$
\left\|T_{t}^{V_{N}} \psi-T_{t}^{V} \psi\right\| \rightarrow 0
$$

as $N \rightarrow \infty$. Summing up the preceding results, we have shown that $T_{t}^{V} \psi, \psi \in L_{2}\left(E^{m}\right)$, exists and is the limit of the $T_{t}^{V_{N}}$ in the strong operator topology (from (2)) for each $t$.

To complete the proof one shows that the range of the resolvent of $T_{t}^{V}$ contains $C_{0}^{\infty}\left(E^{m}\right)$ and thus from the general theory in $\S 22.3$ of [4] we will have that $T_{t}^{V}$ is a strongly continuous (on $[0, \infty)$ ) selfadjoint semi-group of operators on $L_{2}\left(E^{m}\right)$. That the infinitesimal generator of $T_{t}^{V}$ extends $\Delta-V$ on $C_{0}^{\infty}\left(E^{m}\right)$ will follow from the discussion of the range of the resolvent of $T_{t}^{V}$.

REMARK 1. From now on $\Delta-V$ will be used to denote the infinitesimal generator of $T_{t}^{V}$. This notation in general is ambiguous but for most applications $\Delta-V$, with domain $C_{0}^{\infty}\left(E^{m}\right)$, is essentially selfadjoint and thus its use seems justified. (See [5].) 
THEOREM 2. Let $g(x)$ and $h(x)$ be functions which satisfy the condition of Theorem 1. Let $V_{n}(x), n=1,2, \cdots$, be a seguence of real-valued Borel measurable functions on $E^{m}$. Suppose that $g(x) \leqq V_{n}(x) \leqq h(x)$, $n=1,2, \cdots$ and $\lim _{n \rightarrow \infty} V_{n}(x)=V(x)$ except for a set of capacity zero. Then

$$
\lim _{n \rightarrow \infty} T_{t}^{V_{n}}=T_{t}^{V}
$$

in the strong operator topology for each $t$ and $T_{t}^{V}$ is a strongly continuous self-adjoint semi-group whose infinitesimal generator is a self-adjoint extension of $\Delta-V$ considered as a symmetric operator on $C_{0}^{\infty}\left(E^{m}\right)$.

Corollary 3 (Getoor-Rellich). Let $V_{n}, V$ be as in Theorem 2. Denote by $\left\{E_{\lambda}^{n}\right\},-\infty<\lambda<\infty$, the spectral resolution of the identity for the infinitesimal generator of $T_{t}^{V n}, n=1,2, \cdots$, and $\left\{E_{\lambda}\right\},-\infty<\lambda$ $<\infty$, the spectral resolution of the identity for the infinitesimal generator of $T_{t}^{V}$. Then

$$
E_{\lambda}^{n} \rightarrow E_{\lambda}
$$

in the strong operator topology for points of continuity of $E_{\lambda}$.

Corollary 4. Given $\psi \in L_{2}\left(E^{m}\right)$ and $V_{n}(x), V(x)$ as in Theorem 2. Let $\psi_{n}(x, t)$ be the $L_{2}$ solution of Schrödinger's equation

$$
\frac{\partial \psi_{n}}{\partial t}=i\left(\Delta-V_{n}(x)\right) \psi_{n}(x, t)
$$

and $\psi(x, t)$ the $L_{2}$ solution of

$$
\frac{\partial \psi}{\partial t}=i(\Delta-V(x)) \psi(x, t)
$$

where the initial condition is

$$
\psi_{n}(x, 0) \equiv \psi(x, 0) \equiv \psi(x)
$$

Then

$$
\left\|\psi_{n}(x, t)-\psi(x, t)\right\| \rightarrow 0 \text { as } n \rightarrow \infty
$$

uniformly in $t$ for bounded $t$ intervals.

Theorem 5. Let $C_{\delta}$ be an open disc of radius $\delta$ about 0 and let $V(x, \epsilon)$ be a complex-valued function on $E^{m}$ such that

(1) $V(x, \epsilon)$ is real for real $\epsilon$ and is analytic in $C_{\delta}$ for each $x$ except perhaps for a set $Q$ of capacity zero.

(2) $V(x, \epsilon)$ is continuous except for a set of capacity zero for each $\epsilon$ 
(3) $\operatorname{Re} V(x, \epsilon) \geqq g(x)$, where $g(x)$ satisfies the conditions of Theorem 1 .

(4) $|d V(x, \epsilon) / d \epsilon| \leqq h(x)$ for $x \in Q$ and $h(x)$ continuous except for $a$ set of capacity zero.

Then $T_{t}^{V(x, \epsilon)}$ is analytic in $C_{\delta}$ for each $t$.

Corollary 6. Let $\lambda$ be an eigenvalue of $(\Delta-V(x, 0))$ where $V(x, \epsilon)$ satisfies conditions of Theorem 5. (See also, Remark 1.) Suppose $\lambda$ has multiplicity $m, 1 \leqq m<\infty$. Then there exist $m$ real analytic functions $\lambda_{i}(\epsilon)$ and $m$ analytic vector-valued functions $\phi_{i}(x, \epsilon)$ analytic for $-\eta<\epsilon$ $<\eta, \eta$ some number $>0$ and such that

$$
(\Delta-V(x, \epsilon)) \phi_{i}(x, \epsilon)=\lambda_{i}(\epsilon) \phi_{i}(x, \epsilon)
$$

$i=1, \cdots, m$ and $-\eta<\epsilon<\eta$.

REMARK 2. The proof of Theorem 2 is essentially a dominated convergence argument. The proof of Corollary 3 is exactly as in [2], corollary to Theorem 5.2. The proof of Corollary 4 uses the LevyCramer Theorem which concerns the continuity of the Fourier transform of measures. Theorem 5 is a straightforward argument showing the analyticity of an abstract integral of a functional which depends analytically on a parameter $\epsilon$. Corollary 6 is a direct application of a theorem of Rellich and Sz.-Nagy [10, p. 376]. See [11] and [12] for some concrete examples of $V(x, \epsilon)$ to which Corollary 6 applies.

Remark 3. The above theorems extend directly to Schrödinger operators on $L_{2}(R)$ where $R$ is an $m$-dimensional, connected subset of $E^{m}$ with a reasonably smooth boundary $\partial R$. In this case we would require the zero boundary condition on $\partial R$.

Applications and detailed proofs will be given elsewhere.

\section{BibLIOGRAPHY}

1. J. L. Doob, Stochastic processes, Wiley, New York, 1953.

2. R. K. Getoor, Additive functionals of a Markov process, Pacific J. Math. 7 (1957), 1577-1591.

3. - Markov operators and their associated semi-groups, Pacific J. Math. 9 (1959), 449-472.

4. E. Hille and R. S. Phillips, Functional analysis and semi-groups, Amer. Math. Soc. Colloq. Publ. Vol. 31, rev. ed., Amer. Math. Soc., Providence, R. I., 1957.

5. T. Ikebe and T. Kato, Uniqueness of self-adjoint extensions of singular elliptic differential operators, Arch. Rational Mech. Anal. 9 (1962), 77-92.

6. M. Kac, Probability and related topics in physical sciences, Interscience, New York, 1959.

7. T. Kato, Quadratic forms in Hilbert space and asymptotic perturbation series, Tech. Rep., No. 7, Office of Ordnance Research, Univ. of California, Berkeley, Calif., 1955.

8. E. Nelson, Feynman integrals and the Schrödinger equation, Princeton Univ., Princeton, N. J., 1963. (multilith) 
9. D. Ray, On spectra of second order differential operators, Trans. Amer. Math. Soc. 77 (1954), 299-321.

10. F. Riesz and B. Sz.-Nagy, Functional analysis, trans. from 2nd French edition by L. Boron, Ungar, New York, 1955.

11. F. Rellich, Perturbation theory of eigenvalue problems, Lecture Notes, New York University, New York, 1955.

12. E. C. Titchmarsh, Eigenfunction expansions associated with second-order differential equations, Part II, Oxford Univ. Press, New York, 1958.

University of California, Los Angeles

\title{
DISTRIBUTION MODULO 1 AND SETS OF UNIQUENESS
}

\author{
BY J.-P. KAHANE AND R. SALEM $\dagger$
}

Communicated by A. Zygmund, July 6, 1963

A linear set $E \subset(0,1)$ is said to be a set of uniqueness (set $U$ ) for trigonometric expansion if no trigonometric series exists (except vanishing identically) which converges to zero in the set $C E$ complementary to $E$. Following Nina Bary we shall say that $E$ is a set of uniqueness "in the wide sense" (set $U^{*}$ ) if no Fourier-Stieltjes series exists (except vanishing identically) which converges to zero in $C E$. If $E$ is a closed set $U^{*}$ it means (see [1, Vol. 1, pp. 344-359, Vol. 2, p. 160]) that $E$ does not carry any measure whose Fourier-Stieltjes coefficients tend to zero. If $E$ is a closed set $U$ (i.e. of uniqueness "strict sense") it means that $E$ does not carry any measure or pseudo-measure (cf. [2]) with coefficients tending to zero.

Definition. A real sequence of numbers $\left\{u_{\kappa}\right\}_{1}^{\infty}$ will be said to be "badly distributed" modulo 1 if there exists at least one characteristic function $X(x)$ of open interval $\Delta \subset(0,1)$ periodic with period 1 such that

$$
\limsup _{\kappa=\infty} \frac{X\left(u_{1}\right)+\cdots+X\left(u_{\kappa}\right)}{\kappa}<\int_{0}^{1} X(x) d x=|\Delta|
$$

when $|\Delta|$ stands for the length of $\Delta .{ }^{1}$

REMARK. It is easy to see that under this hypothesis there exists a $\Delta$ with rational end-points having the same property.

THEOREM. Let $E \subset(0,1)$ be a linear set such that there exists an infinite sequence of positive integers $\left\{n_{\kappa}\right\}_{1}^{\infty}$ increasing to infinity, with the

$\dagger$ Professor Salem died June 20, 1963, in Paris.

1 The reader will convince himself that all the argument which follows is applicable in the case we suppose $\lim$ inf $>\Delta$. 